\title{
ECONOMÍA ECOLÓGICA: HACIA UNA ECOLOGÍA EN LA ENSEÑANZA DE LA ECONOMÍA
}

\author{
German Rodríguez Gama \\ Pontificia Universidad Javeriana de Colombia
}

\begin{abstract}
Resumen: La Economía Ecológica se presenta como una alternativa en método y forma que da respuesta a las deudas que la economía tradicional ha ignorado históricamente. Los movimientos estudiantiles en el mundo demandan una reforma de la enseñanza de la disciplina que responda a la crisis más grande que enfrentamos como humanidad: la sostenibilidad de la vida.

En este texto se hace un breve recorrido sobre la forma como la economía tradicional ha abordado los temas asociados al medio ambiente, los retos y limitaciones y se presenta a la Economía Ecológica como una propuesta alternativa que se alimenta de la transdisciplinariedad.
\end{abstract}

Palabras clave: Economía ecológica. Educación. Heterodoxia. Naturaleza. Ecología de saberes. 


\title{
Ecological Economics. Towards an Ecology in Teaching of Economics
}

\begin{abstract}
The Ecological Economics is an alternative that respond to the debts that the traditional economics has historically ignored. The students' movements around the world demand a deep reform in the education of economy to attend the greatest crisis we face as humanity: the sustainability of life.

In this text, we briefly review the way in which the traditional economy has approached the issues associated with the environment, the challenges and limitations and presents the Ecological Economics as an alternative that extracts from the transdisciplinarity.
\end{abstract}

Keywords: Ecological Economics. Education. Heterodoxy. Nature. Knowledge ecology.

\section{German Rodríguez Gama}

Economista de la Pontificia Universidad Javeriana de Colombia. Magister en Estudios Internacionales en Paz, Conflictos y Desarrollo de la Universitat Jaume I de España.

Ha investigado y trabajado en temas relacionados con el conflicto armado colombiano, especialmente sobre paramilitarismo, víctimas, derechos humanos y reconciliación y perdón; asimismo, acerca del tráfico de mujeres con fines de explotación sexual en Castellón, España. Recientemente ha investigado la teoría económica heterodoxa o crítica.

Correo electrónico: german-960@hotmail.com; al360968@uji.es 


\section{Introducción}

En el artículo «Bioeconomía y biodesarrollo» escrito por Carlos Eduardo Maldonado en Le Monde Diplomatique se puede leer la que podría ser la definición más certera de nuestra realidad en relación con las crisis: «La crisis no es únicamente financiera o comercial, económica o política. Es de concepción de la vida y de forma de vivir» (2012: 32-33).

Este es justamente el reto más importante al que nos enfrentamos como humanidad y que debe contar con la participación crítica y propositiva de la economía como disciplina, siendo justamente esta la que más ha ignorado el impacto ecológico y los efectos de su actividad, que no tiene que ver únicamente con el objeto de estudio y métodos aplicados, sino que su enseñanza en las facultades del mundo debe responder a esas necesidades que el mundo demanda.

En este texto se pretende mostrar de manera sencilla la responsabilidad existente entre la enseñanza de la Economía como disciplina y su relación con el modelo de desarrollo. Así mismo, en respuesta a las críticas que se le hace a la teoría neoclásica como enfoque hegemónico en relación con las crisis, especialmente la ambiental, se presenta como alternativa epistemológica y metodológica la Economía Ecológica, que forma parte de las corrientes heterodoxas de la economía, junto con las economías feminista, institucional, postkeynesiana, marxista y del desarrollo.

Es importante decir que la economía heterodoxa o crítica, en contraposición a lo que sucede en los enfoques tradicionales, ha procurado compartir luchas y beber de la interdisciplinariedad como un elemento fundamental de su construcción. Por esto, el lector encontrará en este texto que los autores aquí citados no son únicamente economistas, sino que están presentes sociólogos, politólogos y para el caso de la Economía Ecológica se encontrará que esta teoría es alimentada por las leyes de la termodinámica como rama de la Física.

También se verá que esta no es una apuesta nueva, sino que se ha mantenido al margen —o mejor dicho, marginalizada- por parte de las corrientes hegemónicas del pensamiento económico. Sin embargo, sus postulados están presentes en la literatura 
desde los años setenta, en las reflexiones acerca del modelo económico, el prisma con el que analiza los fenómenos socio-económicos, los diagnósticos y propuestas que emite en relación con realidades contextuales; así como las deudas que el sistema económico tiene con la naturaleza, las mujeres, los pobres, las generaciones futuras y las relaciones de poder existentes dentro de un marco institucional. Por ello, se iniciará con un breve recorrido por los contenidos teóricos en microeconomía y macroeconomía para hacer evidente las apuestas de la economía heterodoxa en relación con las deudas y falencias que la teoría tradicional presenta en la enseñanza de la disciplina y su relación con el medio ambiente.

Todo lo anterior ha sido manifestado por movimientos estudiantiles y organizaciones de la sociedad civil que exigen un nuevo enfoque en la enseñanza de la disciplina, por lo que este texto incorpora los aportes epistemológicos de Boaventura de Sousa Santos en relación con la necesidad de un pluralismo de saberes y conocimientos.

Este enfoque plural es una necesidad latente y demandada por los movimientos de estudiantes en el mundo y por los movimientos sociales organizados que abogan por la formación de los estudiantes de economía de pregrado y posgrado con un enfoque interdisciplinar, rescatando la filosofía y la historia que de manera gradual han adquirido un papel secundario y optativo en la formación de los economistas.

\section{La enseñanza de la economía en relación con el medio ambiente}

Las facultades de economía del mundo han seguido la teoría neoclásica como modelo hegemónico de enseñanza de manera dogmática, poco o nada plural y sacrificando espacios fundamentales para la formación del estudiante como la economía política, la historia y la filosofía. En este sentido, la economía ortodoxa - la que ocupa las universidades en gran medida para explicar las relaciones económicas - se ubica por un lado sobre el pensamiento marginalista que alimenta la teoría microeconómica, cuyo razonamiento se da en función del análisis costo-beneficio, y por otro lado, se sitúa sobre un fragmento del pensamiento keynesiano sobre el que se desarrolla la teoría macroeconómica.

Todo estudiante de economía se habrá encontrado en su curso de Introducción o Fundamentos con una definición de la economía que en 1932 formuló Lionel Robbins y que es la constante en los manuales de economía. Se define a la economía como «[...] la ciencia que estudia la conducta humana como una relación entre fines y medios limitados que tienen diversa 
aplicación» (1944: 23) y que se ha convertido en un elemento incuestionable para los estudiantes del mundo.

Siendo este el eje sobre el que se desarrolla la disciplina y que a su vez sustenta la micro y la macroeconomía, no sorprende que los efectos asociados del sistema económico y los impactos ecológicos de los que es responsable, estén sesgados desde su base. Por esto resulta fundamental un cambio de paradigma a la hora de estudiar, cuestionar y proponer alternativas en función de las realidades que como humanidad enfrentamos.

La respuesta en términos microeconómicos a las cuestiones ambientales es la Economía Ambiental, que estudia estos fenómenos sin cuestionar de fondo los parámetros estructurales que desde la teoría tradicional se han legitimado y perpetuado. Siendo así, se establece como elemento de análisis a las externalidades, que son efectos (positivos o negativos) que no están representados en los precios de mercado. Teniendo en cuenta que para la teoría neoclásica y de libre mercado los precios son los reguladores por excelencia, las externalidades son concebidas como fallos de mercado que deben solucionarse internalizando la externalidad (Martínez Alier 2008), un concepto popular en la microeconomía y especialmente en la Economía Ambiental.

Esto último lo que quiere decir es que se deben generar incentivos fiscales o monetarios para que las empresas usen mejores tecnologías. Aquí lo cuestionable es un elemento endógeno en el avance tecnológico para la producción que se conoce como la «Paradoja de Jevons: más eficiencia en el uso de recursos, costos relativos más baratos, por ende mayor uso de recursos» (Martínez Alier 2008: 30) y el conocido efecto rebote resultado de los avances tecnológicos que generan mayor eficiencia en el consumo de energía y materiales que tiene como efecto, en términos absolutos, un mayor uso de energía y recursos (Cansino et al. 2017).

Como segunda medida se propone generar un mercado para los efectos negativos y residuos de la producción, por lo que volvemos a la asignación de criterios monetarios intentando que los precios reflejen todos los efectos de la producción y el consumo. Vale la pena preguntarse, ¿cómo establecer un criterio monetario para compensar a las generaciones futuras por la contaminación del aire o la pérdida de biodiversidad?, ¿cómo valoramos el desplazamiento de comunidades enteras para el desarrollo de proyectos agroindustriales o minero-energéticos?, ¿cuánto cuesta la salud de una persona que se ha visto afectada por el uso de pesticidas industriales? Para ejemplificar lo que queremos decir vale preguntarse, ¿el precio del barril del petróleo refleja los efectos para las generaciones futuras, la contaminación asociada a la extracción, transporte y quema, el desplazamiento de comunidades indígenas o campesinas por las externalidades generadas o la pérdida de sitios sagrados para su cultura? 
Y no únicamente hablamos de la valoración de los efectos negativos sobre los que la Economía Ambiental intenta dar una respuesta sin cambiar la perspectiva marginalista con que analiza los problemas. Hablamos de que la economía tradicional se inscribe metodológicamente en la sostenibilidad débil, dejando ver una de las grandes grietas del pensamiento económico ortodoxo: el cortoplacismo en el pensamiento y sus propuestas.

Suponer que la economía funciona como un ciclo cerrado en donde entran recursos y salen productos, y en el que interactúan únicamente las familias que ofrecen su fuerza de trabajo y las empresas que contratan esa fuerza de trabajo junto con el capital para la producción de bienes y servicios que son consumidos por las familias gracias al salario que devengan, deja por fuera del análisis un sinnúmero de relaciones que se dan en la realidad.

La economía no funciona como un ciclo, sino como parte de un sistema abierto y complejo del que el sistema económico es solo una de las piezas dentro de un complejo engranaje. Siendo más precisos, el sistema económico es un subsistema dentro de uno más grande que es el sistema social que a su vez está dentro del sistema natural (Cansino et al. 2017).

Gráfica 1:

Conexión de sistemas subordinados (Economía Ecológica)

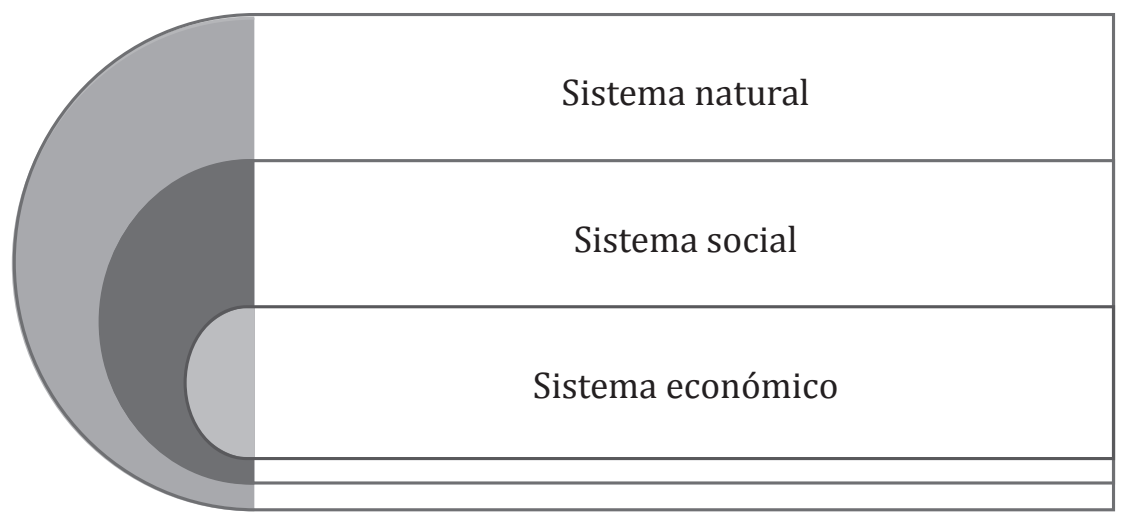

Fuente: Cansino et al. (2017)

En ese sistema abierto y complejo que es la economía, se generan interacciones con los elementos propios e interconexiones con los elementos de los otros sistemas con los que se relaciona. En él intervienen, no únicamente la racionalidad de los agentes, sino un conjunto de leyes, expectativas, incertidumbres, realidades observables y el azar, que actúan y configuran el sistema como un todo (Álvarez Caltapiedra et al. 2012). 
Adicionalmente hay que decir que el sistema económico no produce nada. Y esto puedo resultar perturbador para cualquier estudiante de economía que ha sido formado en la teoría neoclásica porque se nos enseña que las empresas producen y los hogares consumen. Para ser precisos, y este es uno de los aportes de la Economía Ecológica, las empresas no producen, sino que extraen y transforman unos recursos naturales con el propósito de obtener bienes y servicios. Además, de ese proceso de extracción y transformación no solo se obtienen bienes y servicios, sino que se obtienen también residuos que son arrojados a la naturaleza (Álvarez Caltapiedra et al. 2012). De esto hablamos cuando decimos que el sistema económico es abierto, porque recibe recursos y produce bienes, servicios, residuos y donde la naturaleza cumple un doble papel: «[...] la naturaleza es la fuente de los recursos (materia y energía) que alimentan la actividad económica y el sumidero de todos sus desechos (sólidos, líquidos y gaseosos)» (Álvarez Caltapiedra et al. 2012: 289).

En la introducción mencionábamos que la Economía Ecológica bebe de la interdisciplinariedad y aquí se puede observar de manera clara por qué lo decimos. En el párrafo anterior se expresó la idea de que en realidad la economía no produce, sino que extrae y transforma. Recuérdese la primera ley de la termodinámica que establece que la materia y la energía no se crean ni se destruyen, sino que se transforman, una tesis que ha sido llevada a la economía por Georgescu-Rogen. Así mismo, cuando se argumentó que la economía es un sistema abierto, estamos refiriéndonos a la segunda ley de la termodinámica o ley de entropía que «es la más realmente económica de todas las leyes naturales» (Georgescu-Rogen 1975: 788). Aunque resulta difícil de comprender incluso para quienes son físicos, aquí lo definiremos de manera sencilla simplemente con la intención de transmitir la idea.

De la primera ley de la termodinámica se puede llegar a pensar que los recursos son ilimitados y que solo requieren de cierto esfuerzo para su transformación. De ahí la importancia de la ley de la entropía, dado que establece que para el caso de la energía existe una porción que se disipa y no es aprovechable, lo que en esencia es un residuo, tal y como sucede con lo que expresáramos anteriormente para el caso de la producción; la economía extrae o transforma recursos naturales que son convertidos en bienes y servicios y una parte de esos recursos son expulsados en forma de residuos no aprovechables (Georgescu-Rogen 1975).

En macroeconomía el panorama no es mucho más alentador, porque se sigue estableciendo una relación entre crecimiento, desarrollo y bienestar social. El indicador que sigue representando el mejor síntoma de salud de la economía es el Producto Interno Bruto (PIB), aunque como manifiesta Joan Martínez Alier «el cálculo oculta más de lo que enseña» (2008a: 13). Con esto último no solo 
nos referimos a la invisibilización de los efectos ambientales de la producción y el consumo asociado al crecimiento, sino de las deudas que tiene el sistema económico con las demás especies de la naturaleza, las generaciones futuras, las comunidades y sociedades empobrecidas, y las mujeres.

Aquí hablamos no de la economía sino del sistema económico porque este último ha sido perpetuado y legitimado por la naturaleza capitalista que lo rige y por el mismo Estado. A continuación, recurriendo a palabras de Moreno Ceballos citado por Edgar Caro-Ramírez (2016), se establece la relación entre el sistema económico y el Estado; así:

Estado, en sentido pleno es, así, un conjunto complejo de aparatos estatales o no, instituciones privadas, agentes, intelectuales orgánicos, normas, leyes, técnicas de poder, teorías, costumbres, ideología, mecanismos de dominación que cumplen las funciones globales de organizar, justificar y mantener el sistema capitalista en su conjunto, tratando de garantizar su permanencia histórica. Actúa garantizando el mantenimiento en primer lugar de las relaciones de producción en sí mismas y las clases sociales sobre las que se sustenta la propiedad privada y el orden de la sociedad burguesa, haciendo posible la producción y la acumulación capitalista, al tiempo que opaca la dominación y neutraliza o combate el cuestionamiento de los sectores explotados, a través de la mediación ideológica y la violencia (Caro-Ramírez 2016: 179).

Como se ha señalado líneas arriba, existen deudas que se desprenden del sistema económico que comprometen a las generaciones futuras, las especies animales y vegetales, las comunidades empobrecidas y las mujeres. Este último elemento y su análisis, es resultado de la articulación existente con la Economía Feminista que reivindica el papel de la mujer como el agente económico que ha sostenido de manera invisibilizada al capitalismo, resultado de unas condiciones patriarcales sobre las que también se sustenta el mismo sistema.

Aunque muchas otras disciplinas han incorporado en enfoque de género en sus análisis - resultado de la movilización de las feministas académicas y los movimientos feministas - la economía ha sido una de las disciplinas cuya intención de configurar un análisis que reconozca las desigualdades existentes no se ha materializado.

Es por esto que la Economía Feminista no se ha desarrollado dentro de la economía tradicional sino de forma paralela; un antecedente que le ha permitido elaborar y proponer, en método y teoría, un nuevo paradigma que permita deconstruir los conceptos tradicionales que integren dentro del 
análisis a la mujer y su papel en los trabajos domésticos, de cuidados y en la esfera pública (Carrasco 2014), no sin antes cuestionar de manera activa que exista una sub-representación de la mujer en la disciplina y la ciencia que genera un tipo de conocimiento androcéntrico y que ha sido incluida en el análisis económico el establecer que «las mujeres dejan de estar ausentes en la economía, toda cuenta que recientemente se "han incorporado masivamente" - expresión habitualmente utilizada- al mercado laboral» (Pérez Orozco 2005: 49).

Cuando decíamos que la contabilidad nacional oculta más de lo que muestra, también nos referíamos al ninguneo del papel importantísimo de la mujer en la sociedad como sustento de la existencia y el desarrollo de la vida en toda la amplitud del término y que traspasa la esfera económica y se inscribe en la vida familiar y comunitaria, política, cultural y pública. Esto, debido a que los trabajos domésticos y de cuidado no remunerados - pero todos ellos fundamentales para la subsistencia, bienestar y reproducciónno son considerados como trabajo, sino resultado de asignaciones de tareas en función de roles socialmente construidos.

Las deudas sobrepasan la invisibilización de la mujer. Están asociadas a los efectos ambientales de la producción y el consumo en detrimento de la naturaleza, las generaciones futuras, los pasivos ambientales, las sociedades empobrecidas y el comercio ecológicamente desigual. Los dos últimos elementos resultan fundamentales en el análisis, por cuanto los países del norte global son quienes en esencia tienen una responsabilidad mayor por las afectaciones que hoy enfrentamos y quienes, irónicamente, se verán menos afectados. Según estudios de la Comisión Económica para América Latina y el Caribe (CEPAL) y el Banco Mundial, los países empobrecidos y las poblaciones más vulnerables son quienes sufrirán las mayores consecuencias negativas de los eventos climáticos (De la Cuadra 2016) resultado de las dificultades económicas y estructurales que tienen para la adaptación al cambio climático.

No se trata de generar una contabilidad nacional que valore cuánto cuestan en términos monetarios las pérdidas del sistema natural para las futuras generaciones para incluirlos en los precios o establecer impuestos, deudas de los países del norte global, el precio de las especies extintas, los bosques arrasados, las comunidades desplazadas, entre otros.

Intentar contabilizar todas las deudas anteriormente mencionadas en términos monetarios nos llevaría necesariamente a caer de nuevo en el paradigma sistémico del sistema capitalista; un paradigma que al girar en torno al dinero genera problemas irresolubles y que resultan ser el foco de preocupación de las economías modernas, sacrificando, tal y como lo vemos en la enseñanza de la economía, por ejemplo, la naturaleza social de la disciplina 
y del que ha de ser su objeto de estudio. Cuando hablamos del paradigma de la economía, nos referimos a: «la inflación, el desempleo, la producción de bienes (y servicios), la distribución del ingreso y el crecimiento y el desarrollo» (CaroRamírez 2016: 190), que son en esencia problemas monetarios.

Entonces, ¿qué se propone para solucionar las deudas en relación con la contabilidad nacional? Existen propuestas de diferentes sectores de la sociedad civil que apuestan por medidas como el PIB verde o apuestas desde el metabolismo social como la contabilidad de flujos de energía y materiales (MEFA, por sus siglas en inglés), la contabilidad de la apropiación humana de la producción primaria neta (HANPP, por sus siglas en inglés) y la huella ecológica individual (Martínez Alier 2008a).

Sin embargo, aquí es interesante introducir una reflexión de Martínez Alier en relación a la necesidad de tener en cuenta medidas que consideren otros lenguajes de valoración para evitar que la ciencia económica establezca relaciones de poder (de nuevo) que ningunee o desconozca los saberes, paradigmas y conocimientos de las comunidades directamente afectadas. De lo que hablamos es de lenguajes de valoración igualmente legítimos, como los derechos territoriales, la justicia ambiental y social, la subsistencia humana y la sacralidad (Martínez Alier 2008b).

Hay que cambiar de rumbo hacia un nuevo puerto. Se trata de asumir las responsabilidades históricas que se tienen en todos los temas anteriores y los que se nos escapan en este texto. Un puerto que incluya en su análisis $\mathrm{y}$ actuar las interrelaciones que existen entre el sistema natural, social y económico, que parta del reconocimiento de que el crecimiento como objetivo central de las economías se traduce en una mayor demanda de recursos y por lo tanto de residuos; que actúe en función de la sostenibilidad coherente con las capacidades que tiene la biosfera de regeneración y asimilación que garantice la vida y el bienestar de todas las especies; alternativas que incluyan una variedad de lenguajes distintos al dinero, que incluyan el costo real de producción y donde se valoren y reconozcan las verdades de las comunidades; por último, establecer que existen relaciones de poder que actúan directamente en la construcción de las reglas de juego en el sistema económico actual para así generar estrategias de transformación hacia la democratización de los derechos del sistema natural (Caro-Ramírez 2016).

\section{Hacia una ecología en la enseñanza de la economía}

Como hemos visto hasta ahora, existe una hegemonía en forma y método de la enseñanza de la economía y la miopía que se ha mantenido en la disciplina en relación a las demandas que el mundo moderno y su sustentabilidad. 
En el año 2001, estudiantes franceses de grandes universidades y escuelas manifestaron su descontento con el método y la forma en que se les estaba presentando el contenido de la Economía. Es así como publican la Carta abierta de los estudiantes de economía a los profesores y responsables de la enseñanza de esa disciplina, manifestándose en cuatro elementos particulares: salir de los mundos imaginaros y situarse en las realidades que les permita una compresión de los fenómenos económicos y sociales; el uso de las matemáticas como herramienta pero no como fin último en la economía que establece una dicotomía entre lo real y lo ideal; la necesidad del pluralismo en las explicaciones que vienen dados por la interdisciplinariedad; un llamado a despertar frente a las necesidades que aquejan al mundo moderno y sobre los que la economía ha sido miope (Castaño 2001).

En 2011 se dio uno de los episodios que evidenció la organización de los estudiantes y la gesta de un movimiento estudiantil, fue el episodio en el que estudiantes de Harvard se levantaron y salieron de la clase del profesor Gregory Mankiw, uno de los famosos economistas y autor de algunos de los textos guía que más se usan en las facultades de economía para la enseñanza de la disciplina. Esto manifestaron los estudiantes en una carta dirigida al profesor: «Hoy, nos estamos saliendo de su clase, (debido al) sesgo inherente a este curso [...] que adopta una determinada y limitada visión de la ciencia económica, que perpetúa [...] la desigualdad económica en nuestra sociedad» (Maya 2012: 9).

Recientemente, el 17 de diciembre de 2017, con la celebración de los 500 años en que Martín Lutero publicara sus noventa y siete tesis para la Reforma Protestante, se han clavado en las puertas del London School of Economics las 33 Tesis para la Reforma de la Economía por parte de Rethinking Economics y el New Weather Institute, argumentando:

Proponemos estas tesis como un desafío al monopolio intelectual insalubre de la economía dominante. Estos son ejemplos de los defectos de las teorías dominantes, de las ideas que las perspectivas alternativas tienen para ofrecer, y de las formas en que un enfoque más pluralista puede ayudar a que la economía se vuelva tanto más efectiva como más democrática (Maya 2017: 8).

Los ejemplos no son pocos y los resultados ya se empiezan a ver. Ejemplo de ello es el Proyecto CORE «cuyo principal resultado es un manual de acceso abierto que se aproxima a las principales cuestiones económicas a través del diálogo con la historia económica y la visualización y acceso a datos económicos» (De la Villa et al. 2017: 317) y el International Student Iniciative for Pluralism in Economics que agrupa a más de cuarenta y dos asociaciones de 
estudiantes en diecinueve países, algunas organizaciones y organizaciones no gubernamentales (ONG) como Post-Cash Barcelona, Economistas sin Fronteras, Econonuestra y Economía Alternativa, la Asociación de Economía Crítica, la Sociedad de Economía Crítica y espacios de divulgación científica y académica como la Revista de Economía Crítica, Economía Crítica y Crítica a la Economía, y Cuadernos de Economía Crítica.

Aun así, el contenido curricular de las facultades no ha variado mucho y cuando mucho, se han introducido de manera optativa clases que no necesariamente son parte de la formación a la que todo estudiante debe acceder. Son avances que se han desarrollado e implementado mayoritariamente en Europa y Estados Unidos. En el caso de Latinoamérica se registran algunos casos en Colombia, Chile y Argentina de universidades que se han sumado al Proyecto CORE, sin embargo, no es algo generalizado.

Existen una serie de necesidades que requieren de un abordaje técnico, pero que estudien las relaciones del mundo real. Este requerimiento al que los estudiantes se han sumado, se viene gestando por académicos desde principios del siglo XX. Ejemplo de ello es Joan Robinson quien en su artículo La economía, hoy dice: «En teoría económica, los modelos sirven para eliminar las complicaciones no esenciales, a fin de poderse concentrar en el aspecto principal; este modelo solo sirve para eliminar el problema» (2004: 26). El llamado de los estudiantes por la diversidad de enfoques queda suportada por el profesor Alberto Ruiz Villaverde se requiere «innovación en los contenidos» (2016: 13), simplemente se ha tecnificado o mejor, matematizado.

A lo que nos referimos, acudiendo a los aportes que Boaventura de Sousa Santos en relación a las epistemologías dominantes, es que existe una monocultura del saber y del rigor del saber, definida como:

Es el modo de producción de no existencia más poderoso. Consiste en la transformación de la ciencia moderna y de la alta cultura en criterios únicos de verdad y de calidad estética, respectivamente. [...] ser cánones exclusivos de producción de conocimiento o de creación artística. Todo lo que el canon no legitima o reconoce es declarado inexistente. La no existencia asume aquí la forma de ignorancia (De Sousa Santos 2010: 22).

Es así como en contraposición, se propone una ecología de saberes que conforme una epistemología de pensamiento propositivo y pluralista. No se trata de establecer aquí un conocimiento universal y que abarque todos los posibles escenarios de conocimiento, por el contrario, lo que se busca es que 
los conocimientos, saberes y ciencia, interactúen y reconozcan los espacios de ignorancia manifiestos en el pensamiento (De Sousa Santos 2010).

\section{Retos y limitaciones}

Aun cuando cada vez se suman más estudiantes a las organizaciones que procuran por un cambio de enfoque en la enseñanza de la economía, apoyados por cada vez más académicos, las facultades han volteado la mirada frente a estas demandas y decidido mantener el rumbo. Los resultados han sido la tecnocracia y el refinamiento de los modelos ya existentes, sin que existan cambios estructurales que nos permita entender ampliamente y de forma plural las relaciones sociales, históricas, naturales y económicas manifiestas en la sociedad.

La hegemonía del paradigma neoclásico sigue presente y no se pretende la extinción del mismo, pero sí la conversación entre este esquema de pensamiento y alternativas teóricas y metodologías hasta ahora invisibilizadas. En primer lugar, es necesario que la disciplina rescate y reincorpore en su quehacer su naturaleza social estudiando a la sociedad en general y particularmente las relaciones económicas dentro de ella (De la Villa et al. 2017).

La tarea no es despreciable, de hecho, las barreras existentes por la gran especialización matemática que vive hoy la economía, considerada como la economía pura, supone que todo aquello que escapa de ese paradigma sufre el riesgo de no ser considerado lo suficientemente riguroso. También es cierto que difícilmente se dará el salto del escenario en el que estamos ahora a uno totalmente nuevo, por lo que la propuesta es estudiar las relaciones de producción, distribución y consumo, pero ampliando el análisis con los parámetros que se proponen aquí; es decir, teniendo en cuenta los limites biofísicos naturales e introducir en el análisis los impactos ambientales el crecimiento, por ejemplo, en el PIB.

Así mismo, se requiere que la formación de los nuevos economistas esté acompañada de múltiples herramientas de análisis: cualitativas, cuantitativas, comparativa, históricas, geográficas, sociales y naturales. Entablar un diálogo con disciplinas como la antropología, la ciencia política, la filosofía y la ecología, que permita una comprensión amplia y profunda de los fenómenos sociales (De la Villa et al. 2017).

Somos testigos de algunos avances en algunas universidades que han incorporado a su oferta de cursos, algunas clases relacionadas con la Economía Crítica y este podría ser un paso natural en el proceso de transformación. Incorporar, a la vez, un curso que en el largo plazo podría 
traducirse en un currículum completamente nuevo. El problema aquí es que no tendremos largo plazo si mantenemos el rumbo actual. Las medidas deben tomarse con urgencia y responsabilidad para la transformación estructural de las relaciones económicas y con la naturaleza.

\section{Conclusiones}

Hemos visto que existen demandas y una movilización importante de los estudiantes. Existe un despertar y una conciencia que permite que una trasformación necesaria se esté adelantando. Estas exigencias están respaldadas por propuestas concretas, resultado de una reflexión importante con respecto al contenido que les es enseñado en sus respectivas facultades. Esta es una movilización con ideas y no una movilización que se exige sin una brújula que oriente el camino.

Las propuestas las hemos visto en las múltiples manifestaciones, cartas, tesis y un largo etcétera, que exigen y ponen sobre la mesa cuáles son las necesidades que el mundo tiene hoy en materia de economía. Lo que no necesitamos son economistas que sean expertos en modelos relativamente complejos pero que son incapaces de cuestionar y proponer esencialmente soluciones a los problemas reales que enfrentamos como sociedad, y con la capacidad de generar un análisis plural con una comprensión amplia de las relaciones económicas, pero también de las sociales y políticas. No se necesitan economistas que ante una crisis solo atinen a decir que es resultado de un mercado que no es lo suficientemente libre y por lo tanto se necesitan políticas liberalizadoras.

Ya que hemos visto que la crisis real que enfrentamos como humanidad es la sustentabilidad de la vida, entonces la economía como disciplina y las facultades de economía del mundo deben hacer lo propio en relación con ese reto que se nos plantea con aviso de urgencia. A los estudiantes un llamado a no parar con la crítica, el cuestionamiento y la movilización, porque de allí es que surgirán gran parte de los resultados requeridos y las alternativas para el mundo que hemos heredado, hasta el momento con fecha de caducidad. 
ECONOMÍA ECOLÓGICA: HACIA UNA ECOLOGÍA EN LA ENSEÑANZA DE LA ECONOMÍA / GERMAN RODRÍGUEZ GAMA

\section{Referencias}

Álvarez Caltapiedra, S., A. Barceló, Ó. Carpintero Redondo, C. Carrasco Bengoa, Á. Martínez Gonzáles-Tablas, A. Recio Andreu y J. Roca JuSMET (2012). «Por una economía inclusiva. Hacia un paradigma sistémico». Revista de Economía Crítica(14), pp. 277-301.

Cansino, D., y M. Castro (2017). «Economía Ecológica». En Angejo Calderón, A., R. Molero Simarro, A. Bullejos Jiménez y C. Martínez Erades (coord.) Hacia una economía más justa. Manual de corrientes económicas heterodoxas. Madrid: Economistas sin Fronteras, pp. 5-50.

Caro-Ramírez, E. E. (2016). «Economía Ecológica. Paradigmas de la economía». Persona y Bioética, vol. 20, núm. 2, pp. 175-191, Cundinamarca.

CARrasco, C. (2014). Con voz propia. La economía feminista como apuesta teórica y política. Madrid: La oveja roja.

CAstaño, J. (2001). «Discusión francesa sobre la enseñanza de la economía». Cuadernos de Economía, 20 (35), pp. 287-296.

De la CuAdra, F. (2016). «Cambio climático y justicia ambiental. Lo público desde los movimientos, las comunidades y las personas». En Lampis, A. (editor). Cambio ambiental global, Estado y valor público: La cuestión socio-ecológica en América Latina, entre Justicia Ambiental y "Legítima depredación". Bogotá: Universidad Nacional de Colombia, CLACSO, INTEPUCP, pp. 105-120.

De la Villa, L. y R. Molero (2017). «Epílogo». En Angejo Calderón, A., R. Molero Simarro, A. Bullejos Jiménez y C. Martínez Erades (coord.). Hacia una economía mas justa. Manual de corrientes heterodoxas. Madrid: Economistas sin fronteras, pp. 299-326.

De Sousa Santos, B. (2006). «La Sociología de las Ausencias y la Sociología de las Emergencias: para una ecología de saberes». En Renovar la teoría crítica y reinventar la emancipación social (encuentros en Buenos Aires). Buenos Aires: CLACSO. Consejo Latinoamericano de Ciencias Sociales, (pp. 1341).

De Sousa Santos, B. (2010). Descolonizar el saber, reinventar el poder. Montevideo: Trilce.

Georgescu-Rogen, N. (1975). «Energia y mitos económicos». El trimestre económico 42 (168-4), pp. 779-836. Recuperado de: http://www.jstor. org/stable/20856519

Maldonado, C. (2012). «Bioeconomía y biodesarrollo». Le Monde Diplomatique, octubre, pp. 32-33.

Martínez Alier, J. (2008a). «Conflictos ecológicos y justicia ambiental». Papeles de relaciones ecosociales y cambio global (103), pp-11-27.

Martínez Alier, J. (2008b). La crisis económica vista desde la economía ecológica. Ecología Política (36), pp. 23-32. 
MaYA, G. (2012). «Los estudiantes contra Mankiw». Ensayos de Economía (40), pp. 9-11.

MAYA, G. (2017). «33 tesis para la reforma de la economía». Ensayos de Economía (51), pp. 7-11.

PÉrez Orozco, A. (2005). «Economía del género y economía feminista ¿conciliacion o ruptura?». Revista Venezolana de Estudios de la Mujer, 10 (24), pp. 43-64.

RobBins, L. (1944). Ensayo sobre la naturaleza y significación de la ciencia económica. Mexico: Fondo de Cultura Económica.

Robinson, J. (2004). «La economía, hoy». En Etxezarreta, M. Seminario de Economía Crítica TAIFA. Crítica a la economía ortodoxa. Bellaterra, Barcelona: Universidad Autónoma de Barcelona, pp. 23-28.

Ruiz VillaVerde, A. (2016). «Introducción a la economía crítica: Un apunte crítico sobre los contenidos y los manuales de economía». En García-Quero, F. J. y A. Ruiz Villaverde (coord.). Hacia una economía mas justa. Una introducción a la economía crítica. Madrid: Economistas sin fronteras, pp. 7-24. 